\title{
Design and Development of a Comprehensive Software for Risk Management of Pipelines Carrying Natural Gas: Conceptual Design
}

\author{
Payam Amir-Heidari', Mehrzad Ebrahemzadih ${ }^{2}{ }^{*}$, Omid Giahi $^{2}$ \\ ${ }^{1}$ Department of Civil and Environment Engineering, Amirkabir University of Technology, Tehran, Iran \\ ${ }^{2}$ Kurdistan Environmental Health Research Center, Kurdistan University of Medical Sciences, Sanandaj, Iran \\ Email: ${ }^{*}$ emhrzad@yahoo.com
}

Received 20 November 2013; revised 20 December 2013; accepted 2 January 2014

Copyright $@ 2014$ by authors and Scientific Research Publishing Inc.

This work is licensed under the Creative Commons Attribution International License (CC BY).

http://creativecommons.org/licenses/by/4.0/

(c) () Open Access

\begin{abstract}
Nowadays natural gas is the main source of energy in most of the countries. This energy carrier is used as a fuel in residential areas and for automobiles, power stations, and other industrial units. Gathering, transmission, and distribution pipelines carry the $\mathrm{NG}^{1}$ from wellhead to end-users. These pipelines are exposed to various types of risks, such as corrosion, ground movement, thirdparty interference, etc. Regarding the critical role of NG in Iran and the risk of fire and explosion, it is necessary to identify, assess, and manage the risks of these pipelines properly. Since the risks of different sections of NG pipelines are completely different from each other, design, development and use of comprehensive software will be helpful in complex procedure of managing the risks of NG pipelines. In this work, the essential features of such software are surveyed.
\end{abstract}

\section{Keywords}

Risk Management; Natural Gas; Pipeline; “What-If” Review; DMRA; Software

\section{Introduction}

Considering the current technologies available in Iran, pipelines are the best and safest way for NG transportation. These pipelines are made of steel or plastic, and are mostly buried under the ground. Since natural gas is a

\footnotetext{
"Corresponding author.

${ }^{1}$ Natural Gas.
}

How to cite this paper: Amir-Heidari, P., et al. (2014) Design and Development of a Comprehensive Software for Risk Management of Pipelines Carrying Natural Gas: Conceptual Design. Open Journal of Safety Science and Technology, 4, 8-14. http://dx.doi.org/10.4236/ojsst.2014.41002 
flammable and combustible substance, it is a hazard and has the potential to cause harm for people living in their vicinity. On the other hand, in addition to the risk of release and fire or explosion, considering the critical role of natural gas as the main source of energy in the country, it is essential to have a systematic method and a suitable tool for assessing and managing the risks of NG pipelines. A proper risk management process will lead to a decrease in accident rates and in the severity of consequences of accidents.

Without the aid of mathematics and computers, it is hard to manage the risks of a complex system such as the wide system of pipelines carrying natural gas. A well-designed software with all the tools that are needed for risk management of pipelines is the need of all the companies that are in charge of safety of NG pipelines.

Natural gas pipelines are divided into three main categories: [1]

a) Gathering pipelines: that transport gas from a current production facility to a transmission line or main. Plainly speaking, gathering lines are those pipelines that are used to transport natural gas from the production site (wellhead) to a central collection point. They generally operate at relatively low pressures and flow, and are smaller in diameter than transmission lines.

b) Transmission pipelines: These pipelines are used to transport natural gas from their respective gathering systems to refining, processing, or storage facilities. Transmission pipelines also transport refined natural gas to customers, for use or for further distribution. Transmission pipelines are constructed from steel pipe and can range in size from several inches to several feet in diameter.

c) Distribution pipelines: Local distribution companies (LDCs) own and operate natural gas distribution pipelines. They receive natural gas from transmission pipelines and distribute it to commercial and residential endusers. Distribution pipelines are generally smaller in diameter than gas transmission pipelines and operate at reduced pressures. Many gas distribution pipelines are made of plastic pipe rather than steel.

These pipelines pass through different areas such as rural and urban areas, near rivers, highways, railways, deserts, agricultural areas, mountain, etc.

People around the pipeline routes are subject to significant risk from pipeline failure. The hazard distance associated with the pipeline ranges from under $20 \mathrm{~m}$ for a smaller pipeline at lower pressure, up to over $300 \mathrm{~m}$ for a larger one at higher pressure [2].

To assess the risks of the natural gas pipelines, we can divide them into three main classes according to their operating pressure:

$>$ Class 150: pressure $=60 \mathrm{psi}$

$>$ Class 300: pressure $=250 \mathrm{psi}$

$>$ Class 600: pressure $=1000 \mathrm{psi}$

In addition to pressure, the conditions of the areas through which these pipelines pass, the material, thickness, type of cover, and so on may also be different for these classes. Pipelines with different conditions will have different risk levels. So it will be better to survey them separately.

The risk assessment of NG pipelines is not a simple procedure. Even different sections of a single pipeline may have different conditions and different risks.

\section{Results and Discussion}

\subsection{Risk}

Risk is defined as a measure of the probability and severity of an adverse effect [3]. To quantify and determine the level of the risk of an accident, we should estimate:

1. The likelihood of this accident

2. The severity of consequences

When estimated, then the risk can be evaluated from the relation (1):

$$
\text { Risk }=\text { frequency } \times \text { consequence }
$$

This is a simple mathematical formula for the risk. But it may be somewhat difficult to quantify the two variables: frequency and consequences! Safety engineers use some statistical and mathematical formulas to determine these quantities. Then using relation (1) we can calculate the level of the risks.

\subsection{DMRA}

DMRA or the decision matrix risk-assessment technique is a systematic approach for estimating risks, which 
consists of measuring and categorizing risks on an informed judgment basis as to both probability and consequence and as to relative. The combination of a consequence/severity and likelihood range, gives us an estimate of risk (or a risk ranking) [4].

Once the hazards have been identified, the question of assigning severity and probability ratings must be addressed. Eventually, the technique is consummated by the construction of the risk matrix and the decision-making table (Figure 1). The new developed DMRA technique has two key advantages: a) It differentiates relative risks to facilitate decision-making. b) It improves the consistency and basis of decision. Moreover, it is aquantitative (due to risk measuring) and also a graphical method which can create liability issues and help the risk managers to prioritize and manage key risks [4].

Working with a Risk Matrix is very easy. The problem is to design or select a suitable matrix in order to be used for risk ranking and decision making. Every organization may have its special matrix, which may vary with that of another organization.

Once you selected a proper risk matrix and identified the hazards, it is time to estimate the frequency and consequences of those hazards according to the scale provided in the risk matrix. This estimation depends on the experience of the members of risk assessment team, and on technical knowledge and ability of team members to use mathematical techniques and modeling software's. After selecting the likelihood and the consequence of a hazard, the level of the risk will be determined. The level of the risks in a risk matrix may be showed by different colors or by labels. The decision-making table below the risk matrix will inform you about what you should do to control the risks.

\subsubsection{Probability of Failure in the NG Pipelines}

Estimating the likelihood and consequences may need different techniques which depend on the type of systems. Usually historical data are used to determine the probability of NG pipeline failures [5]. The failure probability of various segments of a pipeline may be completely different. The failure rate of a pipe line varies significantly with design factors, construction conditions, maintenance techniques and environmental situation, etc. [6].

The probability of failure in the pipelines is defined as the number of failures per unit length of pipe in one year (1/year. km), assuming uniform conditions along the pipeline section of interest [7]. This probability is not a constant value and depends on various factors.

The factors that are directly involved in probability of failure of low-pressure $\mathrm{CI}^{2}$ pipelines (in gas distribution

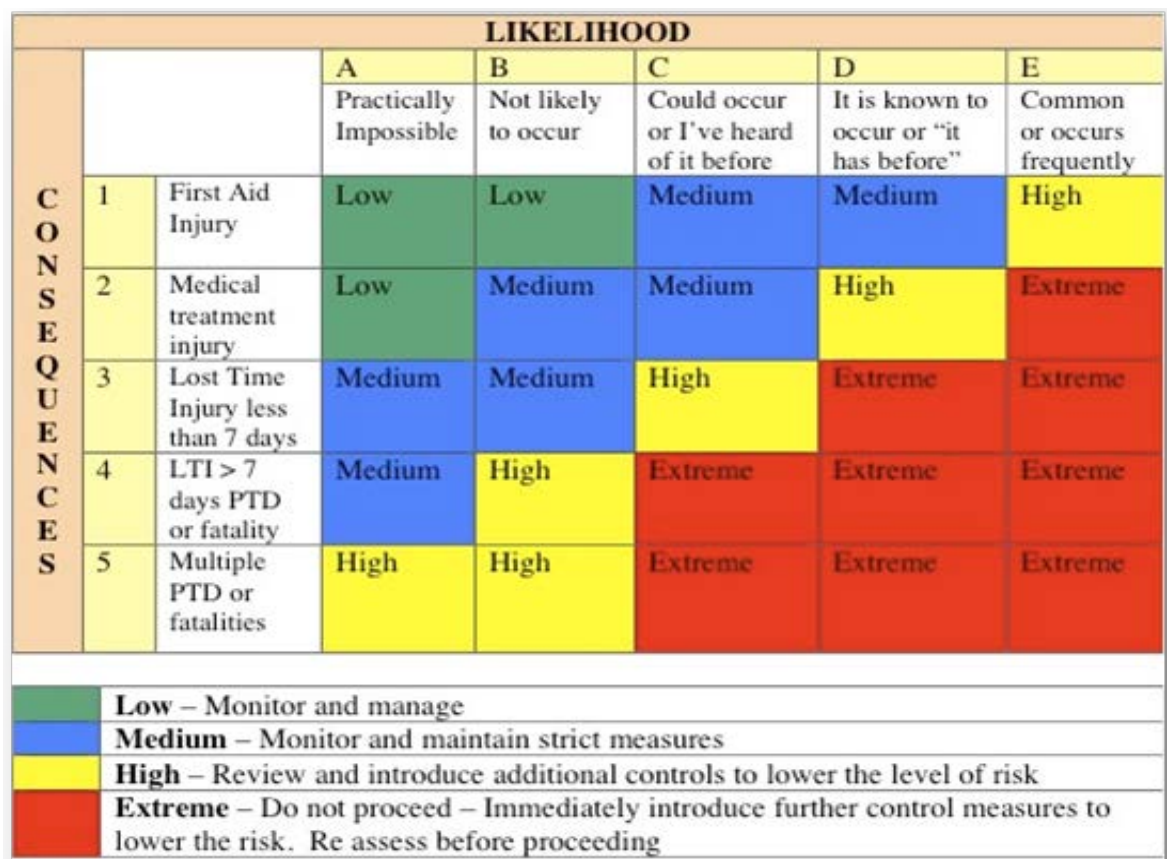

Figure 1. A sample risk matrix [8]. 
network) are: [9]

- intrinsic features of the pipeline-section

-Thickness

-Diameter

-Age

- factors concerning the laying of the pipeline-section

-Depth

-Location

-Ground characteristics

-Type and state of the pavement

-laying techniques

- environmental parameters of the pipeline-section

-Traffic characteristics

-Intensity of underground services

-External temperature and moisture

We can gain some empirical formulas that relate each of these factors to accident rates. These formulas and the historical data together will be a good criterion for estimating the probability of failure of NG pipelines.

\subsubsection{Consequences of the NG Pipeline Accidents}

In order to assess the risk regarding the natural gas pipeline, it is necessary to evaluate probable undesirable consequences resulting from any pipeline leakage or rupture [10].

As a result of an accident in a pipeline a large amount of fuel gas enters into the atmosphere. This release may lead to consequences such as fire or explosion [11] and cause harm to the adjacent people and buildings.

When the released gas intensively mixes with air and forms a persistent vapor cloud, it will lead to a significant flash fire or unconfined vapor cloud explosion. In the case of natural gas pipelines the probability of flash fire is very low, since natural gas is lighter than air, and because of its buoyant nature it cannot form a persistent vapor cloud at the ground level. Therefore the overpressure from unconfined vapor cloud explosion can be ignored. The toxic effect of natural gas leakage can also be neglected [6]. So the consequences of the NG pipeline accidents are usually: thermal radiation of the jet fire and the hazard of confined explosion.

A jet fire, referred to a flame jet, occurs when a flammable chemical is rapidly released from an opening in a container and immediately catches on fire-much like the flame from a blowtorch. Thermal radiation is the primary hazard associated with a jet fire [12]. In the case of natural gas pipelines, the thermal radiation will be the most important hazard of a probable accident.

When the gas is burning at the leakage source, the fire becomes diffusion flame (i.e. jet flame) and poses a threat to the people near the leakage source. The risk of jet flame can be quantitative judged by the measure of thermal radiation.

The other probable consequence of natural gas pipeline accidents is confined vapor cloud explosion. The most basic definition of an explosion is a sudden, intense release of energy that often produces a loud noise, high temperatures, and flying debris, and generates a pressure wave. There are many types of explosions and the causes and effects will vary. We should consider three primary hazards when dealing with an explosion: thermal radiation, overpressure, and hazardous fragments [12].

If the released gas does not catch fire immediately after escaping from a crack or hole, it may travel to buildings and closed places near the leakage point and form a hazardous combustible atmosphere. A delayed spark at this combustible space will lead to confined vapor cloud explosion and sequent outcomes.

Nowadays modeling software's help specialists to simulate these scenarios and determine the threat zones. The outcome model of these software's, such as PHAST or ALOHA, only shows the threat zones and not the severity of consequences! E.g. the output model of this software's for a scenario of jet fire is the same for both a pipeline in desert and in a populated area. In our proposed software these problems all are regarded and the severity of consequences can be directly computed using that.

It should also be considered that in addition to fire and explosion, there is a hazard of formation of gas hydrate and obstruction of the NG pipelines, especially in low temperatures and cold days of the year. All the hazards should be considered and all the risks should be controlled. 


\section{3. "What-If" Plus DMRA}

"What-if" is a qualitative method for Hazard Identification and risk assessment. Although this method is a qualitative method, when combined by DMRA technique, a very suitable method will be gained for risk assessment of NG pipelines.

We suggest the "What-if" frame, because:

- It can be accomplished with a relatively low skill level: The typical What-If review is a basic brainstorming session; all sorts of topics may be randomly addressed as they come to mind. Combined with a checklist format, the review may become simple questions to answer.

- It is fast to implement, compared to other qualitative techniques: Since the "What-if" review is a direct question method possibly from a standardized checklist, the questions can be easily and usually rapidly addressed.

- It can analyze a combination of failures: The option of addressing continuing sequential failures can be investigated to the final outcome.

- It is flexible: It is readily adaptable to any type of process flow or facility. Questions can focus on specific potential failures [13].

After the "What-if" review, the risks can be easily quantified using DMRA technique. Then each risk can be showed by a number, which is an indication of the level of that risk. By comparing these numbers with the criterion, we can determine whether the level of a specific risk is acceptable or not!

Risk criterion is a number that is the boundary for acceptable risks; that is the accidents with a risk greater than the criterion number are not acceptable for us, and extra controls and actions are needed in order to lower the level of those risks. There are some special methods to calculate the risk criterion for a system or organization; however we can use safety manuals and references to find the risk criterion for the system of pipelines carrying natural gas. Risk criteria are different from one organization to another.

We can also use a decision making table to beware of the required actions.

\section{The Process of Risk Management by Our Proposed Software}

Risk management is the culture, process and structure, which come together to optimize the management of potential opportunities and adverse effects [14]. A proper management of risks will assure the safety of the systems.

There is a general procedure for risk management, which is the basis for any work done for management and controlling the risks of hazards in a system or organization. Risk management is a dynamic process, and should be done continuously during the whole life cycle of the systems. Figure 2 shows the main steps of this process.

Although this frame may be the same for all of the systems, the methods or the techniques that are used, could vary from one case to another.

The steps of risk management cycle in our proposed software for NG pipelines are as below:

1) Specify a certain segment of the pipeline as the system to be surveyed. It should be noted that all of the conditions and parameters should be the same for all parts of this segment.

2) Identify the hazards of this segment. It is better to use the "what-if" method to find and identify the hazards. Also preparing and using "Check Lists" is recommended beside the "what-if" review

3) Estimate the probability of failure for one hazard in this segment using historical data and formulas relating the pipeline characteristics and parameters to failure rate.

4) Estimate the severity of consequences and outcomes. This will be done in two steps: first, modeling of the threat zones of predicted scenarios and second, converting these models to severity of accidents, regarding population density, probit ${ }^{3}$, and other empirical formulas.

5) It should be noted that in addition to the risk of fatalities and injuries, other types of risks such as economic risks can also be regarded.

6) Beside the accidents that lead to gas release and fire and explosion, other type of hazards such as the hazard of formation of gas hydrates and pipeline obstruction should be considered.

7) Use a proper risk matrix to determine the level of the risk for the identified hazard using the estimated likelihood and consequence. You may use a present and ready matrix or design and prepare your own specific matrix according the accuracy required.

8) Do this for other identified hazards, other sections, and other pipelines

${ }^{3}$ Probit is the "probability unit". 


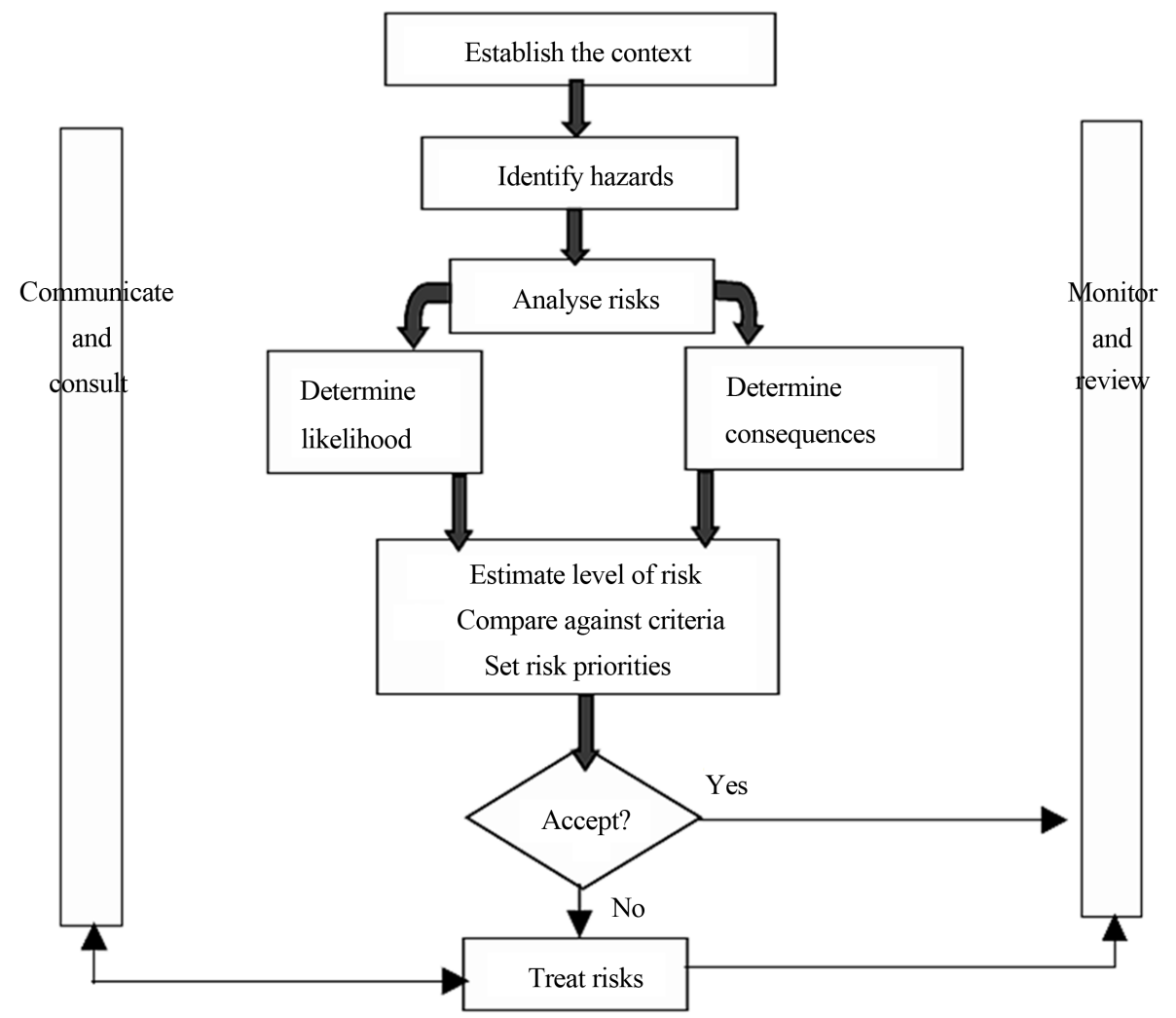

Figure 2. Risk management process [14].

9) Prioritize the risks of these hazards. Risks of higher level should firstly be considered. Perhaps an immediate action may be required for some highly risky hazards.

10)Use a decision making matrix, or consult to identify the proper engineering or administrative controls to lower the level of the risk.

11)Document all of the actions done, using appropriate worksheets designed specifically for your company.

12)Repeat this process from time to time to be certain about the safety of pipelines. The time between two assessments depends on the conditions of the pipelines.

This ten-step procedure will help you to identify, assess, and manage the risks of the system of NG pipelines in a short time and with a low cost. These all will be done by the help of a single software, which is designed as a simple tool for risk management of NG pipelines.

\section{Conclusions}

Considering the critical and strategic role of natural gas in today's countries, it seems necessary to design and develop an appropriate software, which can be used as a comprehensive "Risk Management Tool" to identify, assess and manage the risks of the natural gas pipelines. The essential features of such software are as below:

- This software should be simple and easy to use.

- It can model the consequences of NG pipeline accidents, i.e. the thermal radiation of jet fires and the threat zone of confined vapor cloud explosions.

- The "Historical Data" of NG pipeline accidents in special local areas can be provided in this software to help the users to determine the likelihood of different scenarios (Hint: this data should be updated).

- Regardless of external interference and some unknown causes of NG accidents, some useful data such as material of pipeline, diameter, pressure, thickness, depth of cover, the condition of buried bed, type of protection, and so on, can be entered by user in order to accurately determine the accident rates by the complementary formulas provided in software data base.

- The population density, economic data, and other information can be entered or modified by user, in order to 
determine and analyze the severity of consequences of accidents from the output models of software.

- Once a systematic technique is selected for assessing the risks, the worksheets of this method should be provided in the software in order to help the user to identify the hazards, analyze and rank the risks, give recommendations, and finally document the risk management. In this work, we suggest that the "What-if" method, combined with DMRA, is suitable for risk assessment of natural gas pipelines.

Instead of installing different programs such as PHAST and PHA-Pro, a safety specialist in a NG transportation company can install a single software which is special for the organization that he or she works with. The time will considerably be saved and the desired data will be gained and organized easily.

\section{References}

[1] http://www.phmsa.dot.gov

[2] Jo, Y.-D. and Ahn, B.J. (2002) Analysis of Hazard Area Associated with High Pressure Natural-Gas Pipeline. Journal of Loss Prevention in the Process Industries, 15, 179-188. http://dx.doi.org/10.1016/S0950-4230(02)00007-4

[3] Haimes, Y.Y. (2008) Risk Modelling, Assessment, and Management. 3rd Edition, John Wiley \& Sons Inc., Hoboken.

[4] Marhavilas, P.K. Koulouriotis, D. and Gemeni, V. (2011) Risk Analysis and Assessment Methodologies in the Work Sites: On a Review, Classification and Comparative Study of the Scientific Literature of the Period 2000-2009,” Journal of Loss Prevention in the Process Industries, 24,477-523.

[5] Amir Heidary, P. (2011) Risk Assessment of Natural Gas Pipeline. 3rd Iranian Pipe \& Pipeline Conference, 24-25 May 2011, Tehran.

[6] Han, Z.Y. and Weng, W.G. (2010) An Integrated Quantitative Risk Analysis Method for Natural Gas Pipeline Network, Journal of Loss Prevention in the Process Industries, 23, 428-436. http://dx.doi.org/10.1016/j.jlp.2010.02.003

[7] Jo, Y.D. and Crowl, D.A. (2008) Individual Risk Analysis of High-Pressure Natural Gaspipelines. Journal of Loss Prevention in the Process Industries, 21, 589-595. http://dx.doi.org/10.1016/j.jlp.2008.04.006

[8] http://www.ionmy.com.au/risk-matrix.aspx

[9] Cagno, E., Caron, F., Cipolletti, D., Mancini, M. and Ruggeri, F. (1997) Assessment of the Failure Probability of Underground Pipelines for the Gas Supply in Ametropolitan Environment: A Robust Bayesian Approach (Italian). Technical Report CNR-IAMI, 97-12.

[10] 7th Report of the European Gas Pipeline Incident Data Group, Gas Pipeline Incidents, December 2008, Document No. EGIG 08 TV-B 0502.

[11] Center for Chemical Process Safety (CCPS) (1994) Guidelines for Evaluating the Characteristics of Vapor Cloud Explosions, Flash Fires, and BLEVEs. Wiley-AICHE, New York.

[12] US Environmental Protection Agency Office of Emergency Management (2007) ALOHA User’s Manual. US Environmental Protection Agency Office of Emergency Management, Washington DC.

[13] Nolan, D.P. (1994) Applications of HAZOP and What-If Safety Reviews to the Petroleum, Petrochemical and Chemical Industries. Noyes Publications, Saddle River, 7.

[14] Macdonald, D. (2004) Practical Hazops, Trips and Alarms. Newnes Publications, Oxford. 